\title{
Impact of Retro Rocket Plumes on Upper Stage Aerodynamics during Stage Separation
}

\author{
Oliver M. Hohn ${ }^{1}$ and Ali Gülhan ${ }^{2}$ \\ Supersonic and Hypersonic Technologies Department, German Aerospace Center (DLR), 51147 Cologne, Germany
}

In this paper we report on an extensive investigation of the separation process of the first two stages of a carrier rocket corresponding to VEGA, which employs solid rocket motors. The effect of the plume of first stage retro rockets on upper stage aerodynamics and aerothermal loads is analysed mostly by means of windtunnel testing in the hypersonic windtunnel H2K of DLR Cologne. Aerodynamic coefficients are determined by force measurements. In addition pressure distributions on the upper stage surface and Schlieren images for flow visualization are recorded. Infrared thermography measurements are conducted to determine the effect on aerothermal loads. Different flow conditions are achieved by variation of Reynolds number, retro rocket injection pressure ratio and angle of attack.

\section{Nomenclature}

$\begin{array}{ll}A & =\text { reference area, } \mathrm{m}^{2} \\ C_{p} & =\text { pressure coefficient } \\ C_{l}, C_{m}, C_{n} & =\text { coefficients of roll, pitching and yaw moments } \\ C_{x}, C_{y}, C_{z} & =\text { coefficients of axial, side and normal forces } \\ D & =\text { reference diameter, } \mathrm{m} \\ M & =\text { Mach number } \\ p & =\text { pressure, } \mathrm{Pa} \\ q & =\text { dynamic pressure, } \mathrm{Pa} \\ R e & =\text { Reynolds number } \\ S t & =\text { Stanton number } \\ x, y, z & =\text { Cartesian coordinates, } \mathrm{m} \\ \alpha & =\text { angle of attack, } \\ \Pi & =\text { pressure ratio }\end{array}$

Subscripts:

$\begin{array}{lll}\infty & = & \text { free stream condition } \\ 0 & = & \text { stagnation condition }\end{array}$

$\mathrm{j} \quad=$ retro rocket jet

\section{Introduction}

OLID rocket propulsion is playing an increasingly important role in recent or future space access applications. $\checkmark$ Especially for carrier rockets, several actual or proposed concepts such as Ares $\mathrm{I}^{1}$, Super Strypi, Liberty, Athena or VEGA $^{2}$ use solid rocket motors as main engines for the first stage in sequential staged systems, as opposed to parallel staged systems like the Space Shuttle, Ariane or Delta rockets, where they are employed only in boosters mounted at the sides of the main first stage which uses liquid propellants.

\footnotetext{
${ }^{1}$ Research Scientist, Supersonic and Hypersonic Technologies Department, Institute of Aerodynamics and Flow Technology, German Aerospace Center (DLR), Linder Höhe, 51147 Cologne, Germany. AIAA Member.

${ }^{2}$ Head of Department, Supersonic and Hypersonic Technologies Department, Institute of Aerodynamics and Flow Technology, German Aerospace Center (DLR), Linder Höhe, 51147 Cologne, Germany. AIAA Member.
} 
The use of solid rocket propulsion in a sequentially staged launcher poses additional challenges on the separation process. Generally, the extent of the impact the retro rocket plume has on upper stage aerodynamics depends on their thrust, which in turn is designed the meet the safe thrust level needed to avoid a crash scenario of the two stages. As solid rocket motors cannot be shut down in an actively controlled fashion, the residual thrust they still deliver when separation takes place is much higher as compared liquid rocket engines. Consequently, in order to achieve sufficient deceleration of the lower stage, retro rockets need to be comparably strong and cause more interference to the flowfield of the upper stage. In the case of VEGA, CFD-analysis showed that the vast extent of the plume during retro rocket firing can cause the flow around the upper stage to almost completely separate and the formation of a very complex flow pattern around the upper stage 3,4 . Similar investigations have been performed on the Ares I rocket $^{1}$ and with a generic conical windtunnel model ${ }^{5}$. They also showed strong impact of the retro rocket plumes on the flowfield around the upper stage.

Within the frame of the ESA TRP "Launcher Stage Separation and Plume Interaction Validation", an extensive windtunnel campaign was conducted for a systematic study of the separation process of such a launching system, where the VEGA rocket was used as the reference for the geometry and the flight trajectory. To simulate the retro rocket jets, highly pressurized air was injected at different pressure ratios. Reynolds number and angle of attack were varied for different flow conditions. The model was designed generic with different modules for the upper stage to allow for different types of measurements. Thus it is possible to determine aerodynamic coefficients by force measurements, pressure distributions and aerothermal loads by infrared thermography.

\section{Experimental Setup}

\section{A. Wind Tunnel and Test Conditions}

The experiments have been conducted in the Hypersonic Windtunnel H2K of DLR Cologne. This facility is a blow down wind tunnel using contoured axisymmetric nozzles for fixed Mach numbers with an exit diameter of 600 $\mathrm{mm}$ for Mach numbers of 5.3, 6, 7, 8.7 and 11.2 at Reynolds numbers in the range of $2.5-20 \cdot 10^{6} \mathrm{~m}^{-1}{ }^{6}$. Depending on the flow condition, test durations of up to $35 \mathrm{~s}$ can be achieved. A sketch of the H2K is shown in Figure 1.

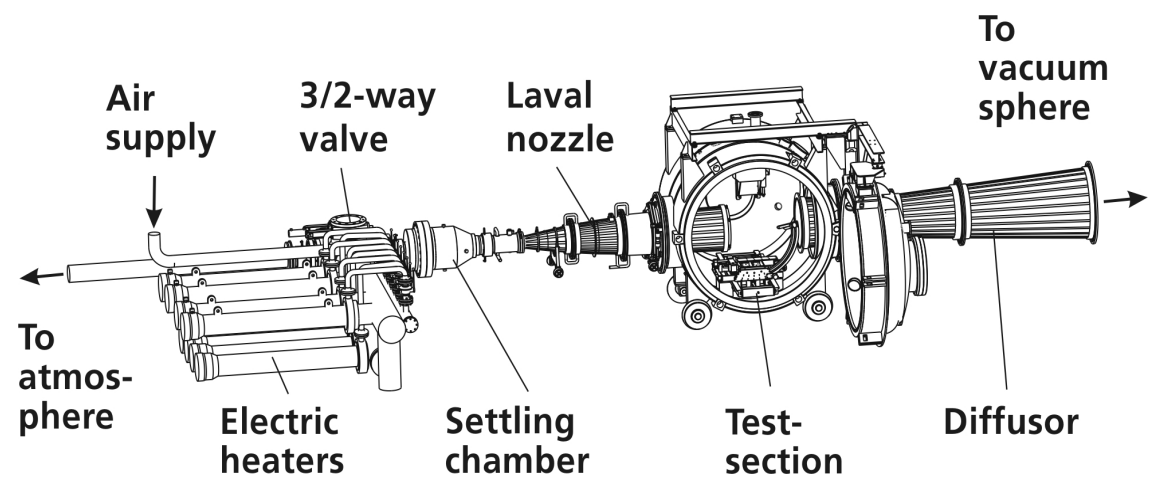

Figure 1: Schematic drawing of the hypersonic windtunnel H2K

Table 1 shows the freestream and retro rocket flow condition present at the trajectory point of first stage separation in real flight. Table 2 lists the windtunnel flow conditions WT 1 and WT 2 used in the experiments. Table 3 presents the two retro rocket flow conditions RR 1 and RR 2, which result from the use of different reservoir pressures. In combination with each windtunnel condition, different retro rocket pressure ratios are achieved. Condition 1 together with RR 1 allows for the pressure ratio to be maximized, whereas condition 2 allows for better replication of the flight condition by meeting Reynolds number similarity. As the pressure ratio is the most deciding parameter in this study, the combination of condition WT 1 and retro rocket condition RR 1, which yields a pressure ratio of $\Pi_{0, \mathrm{j}}=15,000$ was used for the majority of tests. During some tests, the retro rocket pressure ratio was varied to examine the changes the plume induces in the flowfield, as the injection pressure increases. In most tests, however, the pressure was increased to the desired pressure level as fast as possible and the behaviour at different angles of attack was investigated. This was done by conducting a sweep from $\alpha=0^{\circ}$ to $\alpha=-6^{\circ}$, then up to $\alpha=+6^{\circ}$ and back down to $\alpha=0^{\circ}$, at a sweep rate of $\Delta \alpha=1^{\circ} \mathrm{s}^{-1}$. An assessment on the influence of the sweep rate on the results was conducted as well by doubling the sweep rate during one test run, but did not yield to have any effect. 


\begin{tabular}{l|c|c}
\hline Flow parameter & freestream & retro rocket flow \\
\hline Mach number & $M_{\infty}=5.37$ & $M_{j}=3.5$ \\
\hline stagnation pressure & $p_{t 0}=143.5 \mathrm{kPa}$ & $\mathrm{p}_{j}=10.56 \mathrm{MPa}$ \\
\hline stagnation temperature & $T_{t 0}=1761 \mathrm{~K}$ & $T_{j}=290 \mathrm{~K}$ \\
\hline freestream pressure & $p_{\infty}=178 \mathrm{~Pa}$ & $p_{\infty, j}=87 \mathrm{kPa}$ \\
\hline freestream temperature & $T_{\infty}=260 \mathrm{~K}$ & $T_{\infty, j}=449 \mathrm{~K}$ \\
\hline ratio of specific heats & $\gamma_{\infty}=1.4$ & $\gamma_{j}=1.2$ \\
\hline Reynolds number & $R e_{m}=2.5 \cdot 10^{5} \mathrm{~m}^{-1}$ & \multicolumn{2}{|c}{$\Pi_{0, j}=p_{0, j} / p_{\infty}=59325$} \\
\hline Pressure ratio & \multicolumn{2}{|c}{} \\
\hline
\end{tabular}

Table 1: Freestream and retro rocket flow conditions at staging in real flight

\begin{tabular}{l|c|c}
\hline Flow parameter & WT 1 & WT 2 \\
\hline Mach number $M_{\infty},-$ & $5.29 \pm 0.03$ & $5.31 \pm 0.03$ \\
\hline stagnation pressure $p_{t 0}, \mathrm{kPa}$ & 300 & 1000 \\
\hline stagnation temperature $T_{t 0}, \mathrm{~K}$ & 390 & 530 \\
\hline freestream pressure $p_{\infty}, \mathrm{Pa}$ & 400 & 1341 \\
\hline freestream temperature $T_{\infty}, \mathrm{K}$ & 58.9 & 80,1 \\
\hline dynamic pressure $q_{0}, \mathrm{kPa}$ & 7.87 & 26.37 \\
\hline Reynolds no. $\operatorname{Re}_{\infty, m}, 10^{6} \mathrm{~m}^{-1}$ & 4.74 & 9.98 \\
\hline
\end{tabular}

Table 2: Windtunnel flow conditions

\begin{tabular}{|c|c|c|c|}
\hline \multicolumn{2}{|c|}{ Flow parameter } & RR 1 & RR 2 \\
\hline \multicolumn{2}{|c|}{ stagnation pressure $p_{0, j}, \mathrm{MPa}$} & 6 & 1.8 \\
\hline \multirow{2}{*}{ pressure ratio $\Pi_{0, j}$} & for WT 1 & 15,000 & 4,500 \\
\hline & for WT 2 & 4,500 & 1,342 \\
\hline \multicolumn{2}{|c|}{ exit Mach number $M_{e x, j}$} & 3.60 & 3.60 \\
\hline \multicolumn{2}{|c|}{ stagnation temperature $T_{0, j}, \mathrm{~K}$} & 290 & 290 \\
\hline \multicolumn{2}{|c|}{ mass flow per RR, $\mathrm{kg} \mathrm{s}^{-1}$} & 0.021 & 0.0063 \\
\hline \multicolumn{2}{|c|}{ ratio of specific heats $\gamma_{j}$, - } & 1.4 & 1.4 \\
\hline
\end{tabular}

Table 3: Retro rocket flow conditions

\section{B. Windtunnel Model}

The geometry corresponds to the VEGA launcher in the scale 1:40. It is a four stage rocket with two cylindrical sections as the first and second stages and a hammerhead nose section containing the payload. The hammerhead and first section are larger in diameter than the second stage by approximately $37 \%$ and $58 \%$, respectively. A conical flare is used for the transition from the second to the first stage. The stages are separated upstream of this flare and the retro rockets are located about in the middle of the flare. The retro rockets injection angle is $11.4^{\circ}$. For the windtunnel model, it is split into two modules: an injection module, which represents the first stage and an upper stage module comprising the remaining three stages and the hammerhead. Both modules are mounted to the strut. The layout and most important dimensions of the model are sketched in Figure 2. Figure 3 shows a photograph of the experimental setup with the model mounted in the $\mathrm{H} 2 \mathrm{~K}$ test section.

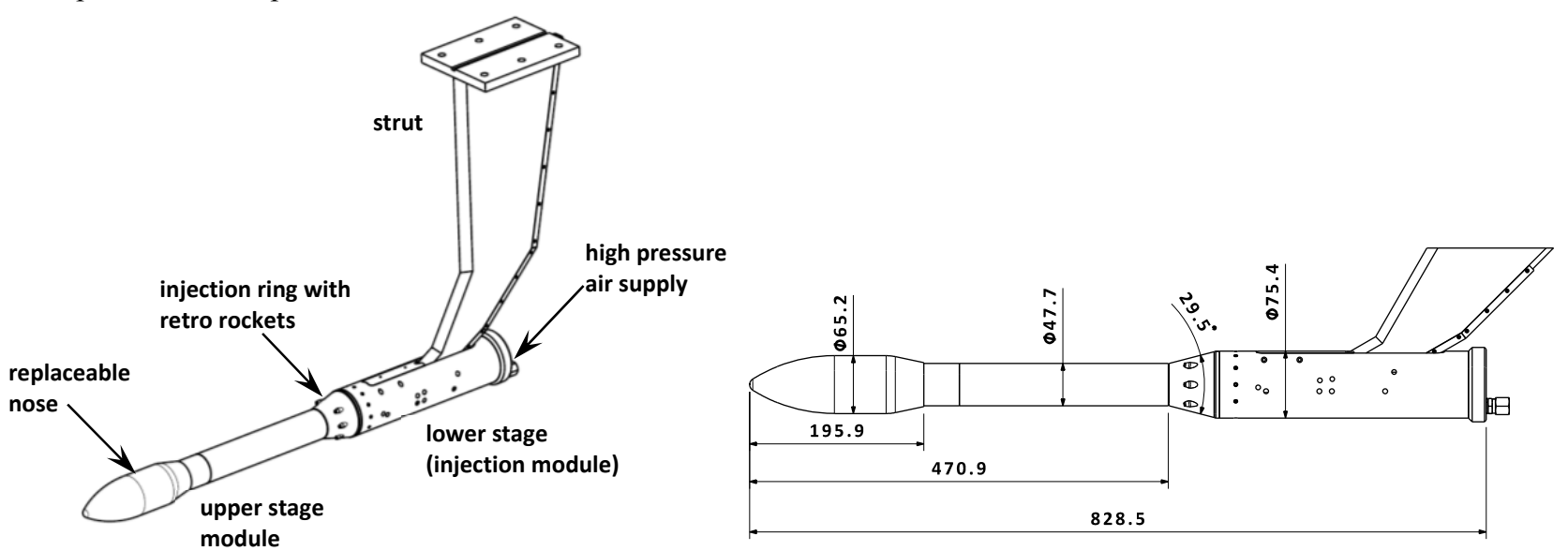

Figure 2: Basic layout and major dimensions of the windtunnel model 


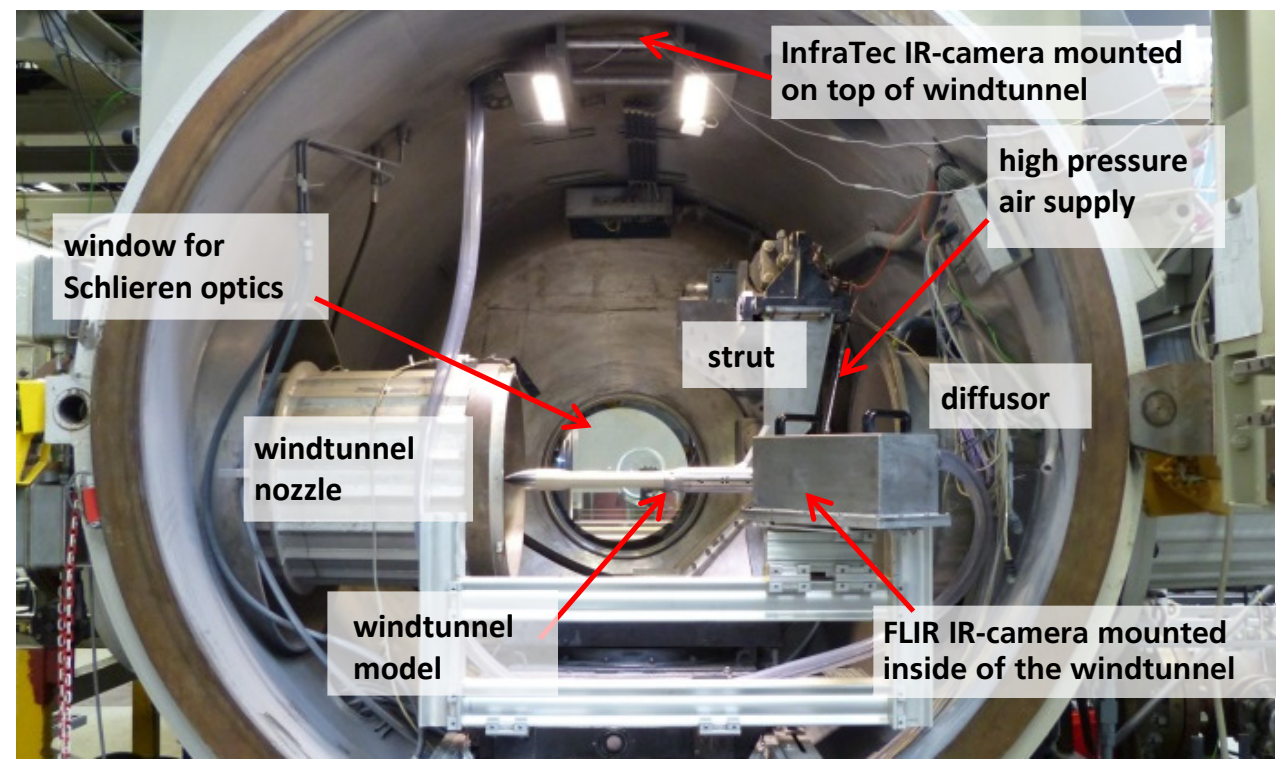

Figure 3: Experimental setup in the $\mathrm{H} 2 \mathrm{~K}$ test chamber

\section{Measurement Techniques}

Three different versions of the upper stage module are available for different types of measurements, i.e. force, pressure and infrared thermography measurements, in order to determine aerodynamic coefficients, pressure distributions and surface heat flux distributions. The complete upper stage is replaced each time a different type of measurement is conducted. In addition, flow visualization is achieved by Schlieren imagery.

\section{A. Flow visualization}

A coincidence or two-pass Schlieren optic is installed at the H2K hypersonic windtunnel facility for flow visualization. To record the images, a Photron Fastcam SA-X high speed camera was used.

\section{B. Measurement of aerodynamic coefficients}

The aerodynamic coefficients are determined by force measurements with a DLR-type six component strain gauge. The maximum loads and accuracies of the balance are:

- Forces:

○ $Z=250 \mathrm{~N}$, accuracy $<0.1 \% \mathrm{FS}$ (full scale)

○ $Y=100 \mathrm{~N}$, accuracy $<0.1 \% \mathrm{FS}$ (full scale)

○ $X=100 \mathrm{~N}$, accuracy $<0.5 \%$ FS (full scale)

- Moments:

○ $M=1250 \mathrm{Nm}$, accuracy $<0.1 \%$ FS (full scale)

○ $N=500 \mathrm{Nm}$, accuracy $<0.1 \%$ FS (full scale)

○ $L=4 \mathrm{Nm}$, accuracy $<0.1 \% \mathrm{FS}$ (full scale)

All forces and moments are given in a body-fixed coordinate system which is displayed in Figure 4. The results are presented as force and moment coefficients where the diameter of the cylindrical section of the upper stage was taken as reference diameter: Prior to the test campaign, the balance was calibrated on a gauging facility.

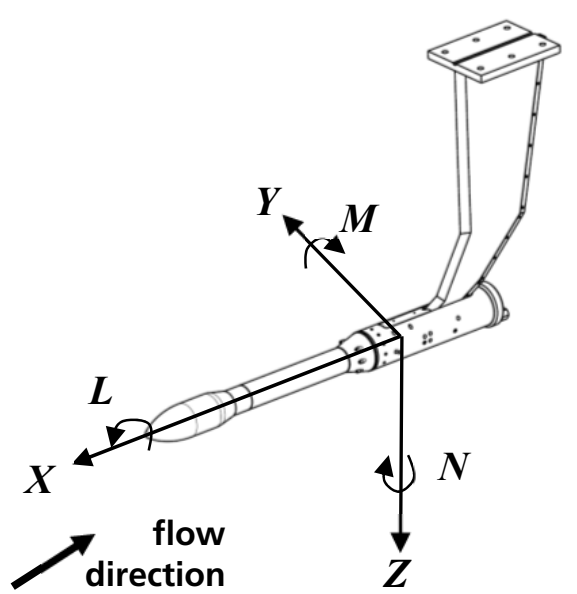

Figure 4: Coordinate system for force measurements

\section{Pressure measurements}

The pressure measurement module contains seven static and instationary pressure probes each, which are distributed equally spaced in streamwise direction along the cylindrical section of the upper stage on opposing sides of the model. A Pressure Systems Inc. 8400 PSI system ${ }^{7}$ for stationary and Kulite XCQ-080 pressure transducers ${ }^{8}$ for instationary measurements are used. Results are presented in terms of dimensionless pressure coefficients. 


\section{Evaluation of wall heat fluxes with infrared thermography}

In order to determine wall heat fluxes, the time wise development of the surface temperature of a upper stage module, where the main body is made of PEEK, is recorded with two infrared cameras, an InfraTec ImageIR $8300^{9}$ mounted on the top of the windtunnel and a FLIR Systems ThermaCam SC $3000^{10}$ mounted inside of the windtunnel in an aluminium housing, as can be seen in the photograph in Figure 3. Thus, the model can be seen from the top, which corresponds to the wind- and leeward sides in the case of negative and positive angle of attack, respectively, and also from the side shifted to the rear. The cameras were calibrated in a test in a test setup similar to the windtunnel setup by a black body. The maximum errors of measured temperatures are $\pm 2 \mathrm{~K}$ or $2 \%$, whichever value is higher, for the FLIR camera ${ }^{10}$ and $\pm 1 \mathrm{~K}$ or $1 \%$ for the InfraTec camera ${ }^{9}$.

The recorded surface temperature distribution is used as the boundary condition for calculating the heat fluxes to the sidewall by evaluating the thermal energy balance of a solid volume:

$$
\rho(T) \cdot c(T) \frac{\partial T}{\partial t}=\nabla(\lambda(T) \nabla T)
$$

Assuming that lateral heat fluxes can be neglected due to the very low heat conductivity of PEEK and accounting for temperature dependent material properties, this transforms into the nonlinear one-dimensional heat equation normal to the wall:

$$
\frac{\partial T}{\partial n}=a(T) \cdot \frac{\partial^{2} T}{\partial n^{2}}+b(T) \cdot\left(\frac{\partial T}{\partial n}\right)^{2}
$$

with the thermal diffusivity

and

$$
a(T)=\frac{\lambda(T)}{\rho(T) \cdot c(T)}
$$

$$
b(T)=\frac{\frac{d \lambda(T)}{d T}}{\rho(T) \cdot c(T)}
$$

Equation (2) is then solved by an explicit finite difference scheme in order to calculate the temperature gradient in the normal direction inside the wall. From this, the wall heat flux can be calculated by the Fourier law:

$$
\dot{q}_{W}=\left.\lambda\left(T_{y=0}\right) \cdot \frac{\mathrm{d} T}{\mathrm{~d} y}\right|_{y=0}
$$

The convective heat flux can then be calculated from the heat flux balance on the surface:

$$
\dot{q}_{c o n v}=\dot{q}_{r a d}+\dot{q}_{W},
$$

where the radiative heat flux is calculated with the Stefan-Boltzmann law

$$
\dot{q}_{\text {rad }}=\varepsilon \cdot \sigma \cdot\left(T_{y=0}^{4}-T_{a m b}^{4}\right),
$$

assuming that the ambient temperature stays constant during the tests. A more detailed description of the evaluation method is given by Henckels and Gruhn ${ }^{11}$. Once the convective heat flux $\dot{q}_{c o n v}$ has been determined, the dimensionless Stanton number can be determined by Eq. (8), with the recovery temperature $T_{r e c}$ calculated by Eq. (9).

$$
\begin{aligned}
& S t=\frac{\dot{q}_{c o n v}}{\rho_{\infty} \cdot u_{\infty} \cdot c_{p, a i r} \cdot\left(T_{r e c}-T_{w}\right)} \\
& T_{r e c}=\left(1+r \frac{\gamma-1}{2} \cdot M_{\infty}^{2}\right) \cdot T_{\infty}
\end{aligned}
$$

Commonly, a recovery factor of $r=0.9$ is used for the flow conditions encountered in the wind-tunnel tests. However, because of the sensitivity of the Stanton number when the wall temperature is close to the recovery temperature (when the denominator approaches zero), it is suggested to use a recovery factor of $r=1$ for the Stanton number in order to allow for a better comparability of the results from different investigations ${ }^{12}$. Therefore, a recovery factor of $r=1$ has been used for the Stanton number evaluation in this study. The accuracy of the results is estimated to be within $\pm 5 \%$ for calculated heat fluxes and $\pm 20 \%$ for the Stanton number.

\section{Results}

\section{A. Cases without injection}

\section{Reynolds number influence}

Lower Reynolds numbers generally result in thicker boundary layers which are more likely to separate. Furthermore, the boundary layer for condition WT 1 is likely to be laminar while transition to turbulent boundary layer is expected to occur for condition WT 2 on the upper stage surface. These assumptions are confirmed by the Schlieren photographs for both windtunnel conditions in Figure 5 and the Stanton number distribution in Figure 6. 


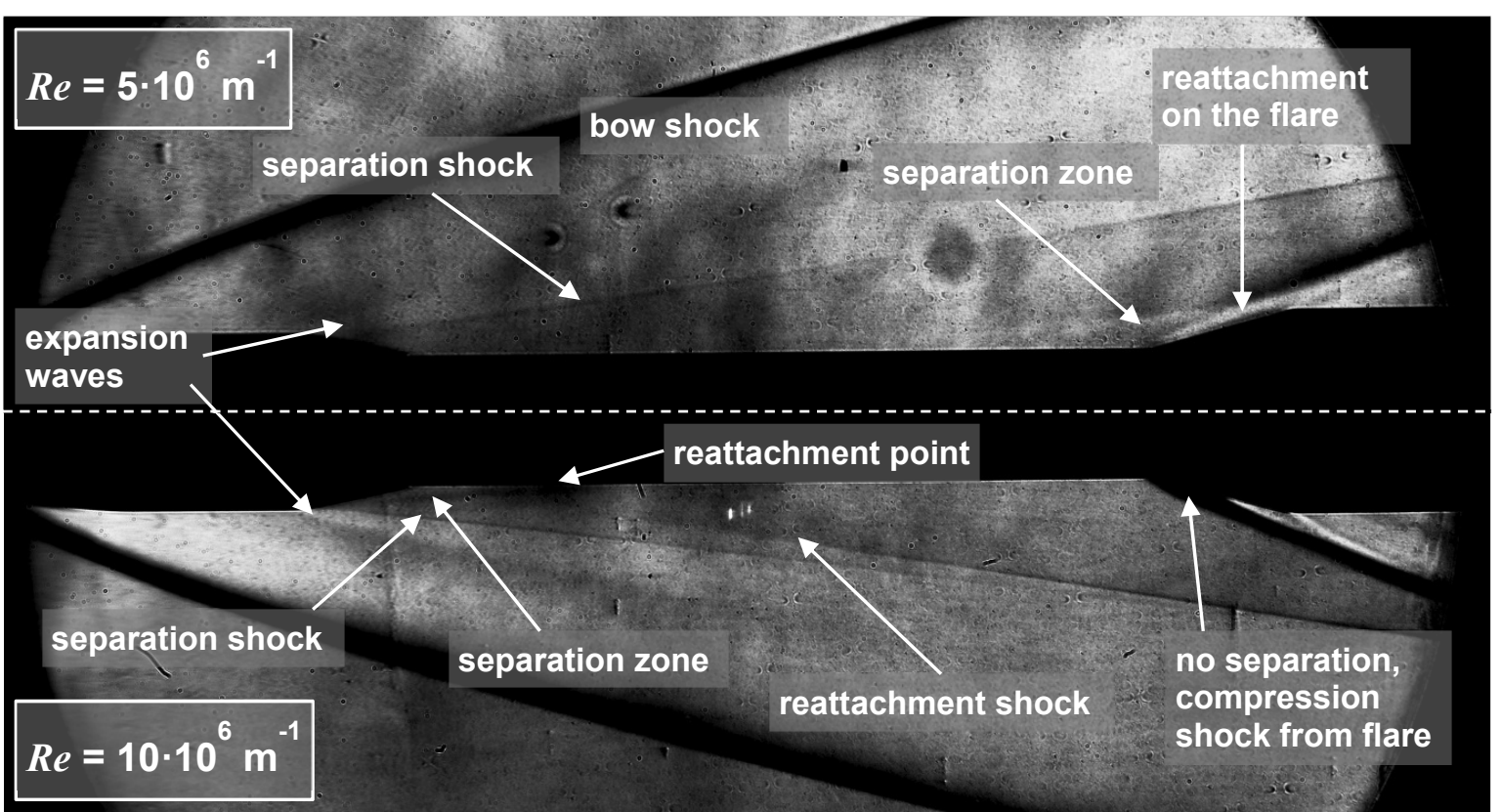

Figure 5: Schlieren images for low (top) and high (bottom) Reynolds number conditions without injection

The flow on the flare of the hammerhead separates just downstream of the expansion corner for both conditions as the separation shocks and the decrease in Stanton number on the flare clearly indicate. While the extent of the separation cannot be determined for condition WT 1, the whole structure of the separated area, i.e. the boundary of the separation zone, the reattachment point and shock are well visible for condition WT 2. However, also for condition WT 1, the flow has to reattach at some point as a second separation zone exists upstream of the first stage flare, which reaches up to the expansion corner at the end of the flare and extends upstream to about the centre of the cylindrical section of the upper stage. The decrease in Stanton number in this area that is visible in Figure 6 is also caused by the separation. The increase towards the downstream end, however, is assumed to be induced by part of the recirculating flow of the separation bubble entering the cavity in between the two stages. For condition WT 2, no separation is observed at the flare of the first stage, but a strong compression shock is induced by the flare. The increase of Stanton number in the downstream half of the upper stage indicates that transition is occurring.

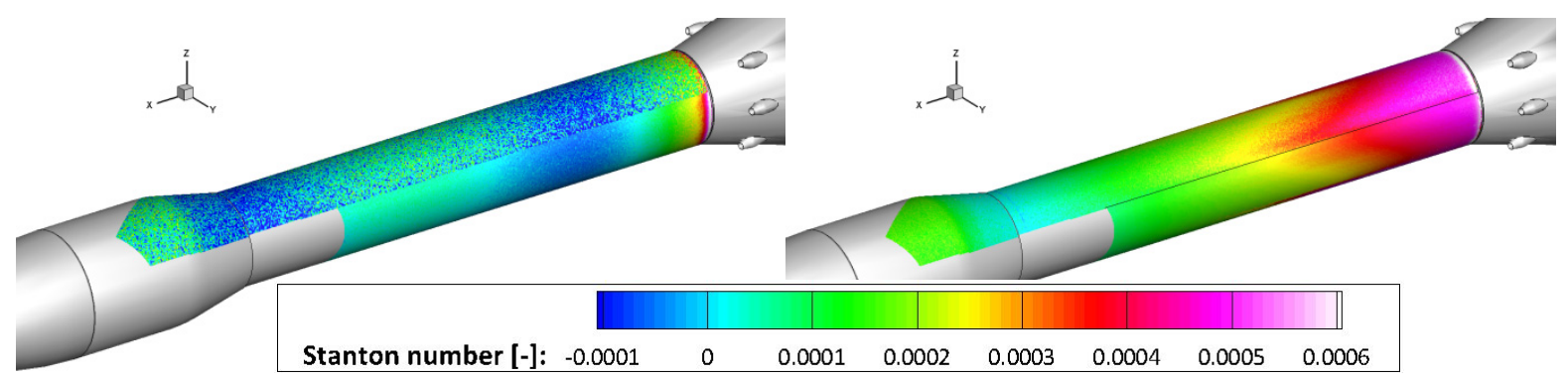

Figure 6: Stanton number distributions for cond. WT 1 (left) and cond. WT 2 (right) without injection

\section{Angle of attack}

Schlieren images for both windtunnel conditions and $\alpha=5^{\circ}$ are displayed in Figure 7. These were recorded during an alpha-sweep as described in section II. At this angle, the differences in the flow structure of both Reynolds numbers are rather small. Especially on the windward side, they look nearly identical, as there is no separation, but expansion at the start of the hammerhead flare and rather strong compression shocks at the beginning of the cylindrical part of the upper stage and the first stage flare. However, the behaviour while changing the angle is slightly different. While for condition WT 1 the separation bubbles simply diminish, for the higher Reynolds number of condition WT 2, a separation zone at the first stage flare, where there was no separation for $\alpha=0^{\circ}$, appears for condition WT 2 at $\alpha \approx 1^{\circ}$ and quickly disappears again when $\alpha$ is further increased. This behaviour is not influenced by

6

American Institute of Aeronautics and Astronautics 
hysteresis. As the model is shifted back to $\alpha=0^{\circ}$ from the turning points at $\alpha= \pm 6^{\circ}$, these separation bubbles appear again around $\alpha= \pm 1^{\circ}$ and disappear at $\alpha=0^{\circ}$.

On the leeward side, when the angle of attack is increased, the separation zones for condition WT 1 become bigger until they connect so that the flow is completely separated at this side. For the higher Reynolds number of condition WT 2, the separation zone at the hammerhead flare becomes larger and a second separation zone appears upstream of the lower stage. The structure of theses separation zones, i.e. the separation and reattachment points are visible quite well.

The differences in the way the flow structure changes on the windward side are responsible for the deviations in the curves of aerodynamic coefficients shown in Figure 8. As it would be expected, the drag of the vehicle, which corresponds to the negative axial force coefficient $-C_{x}$, increases quite steadily and symmetrically with both positive and negative angle of attack. The normal force, as it would be expected, shows a steady, nearly linear decrease when going from negative to positive angle of attack.

For the higher Reynolds number, however, the build-up and diminishing of the separation bubbles at the lower stage flare causes a decrease in drag when the model is shifted from $\alpha=0^{\circ}$ to either positive or negative angle of attack, until about $\alpha= \pm 1^{\circ}$. After that, it increases again. However, the increase is less steep and the values at the maximum angles of $\alpha= \pm 6^{\circ}$ are smaller. These changes also result in differences in the curves of the axial force. They also are quite steady and show a nearly linear increase from negative to positive angle like it is the case for the low Reynolds number. In the region of $-1^{\circ}<\alpha<1^{\circ}$, however, the gradients for the higher Reynolds number are higher but then a region follows up to $\alpha= \pm 3^{\circ}$ where the gradients are lower. At this point the values for both Reynolds numbers are almost equal. From this point, the curves for the higher Reynolds number are a bit steeper.

The angle of attack influence on the Stanton number distribution is displayed in Figure 10 for condition WT 1. The streak with high Stanton number along the centreline of the leeward side indicates the formation of a pair of counter-rotating vortices by the flow circulating around the cylindrical part which causes strong heating in this area. Apart from this, the Stanton number on the leeward side is lower than for $\alpha=0^{\circ}$ because of the flow separation. Because of the expansion on the flare, and on the leeward side also because of the separation, the Stanton number decreases on both sides. At the windward side of the cylindrical section the Stanton number is much higher due to the heating of the surface by the oncoming flow.
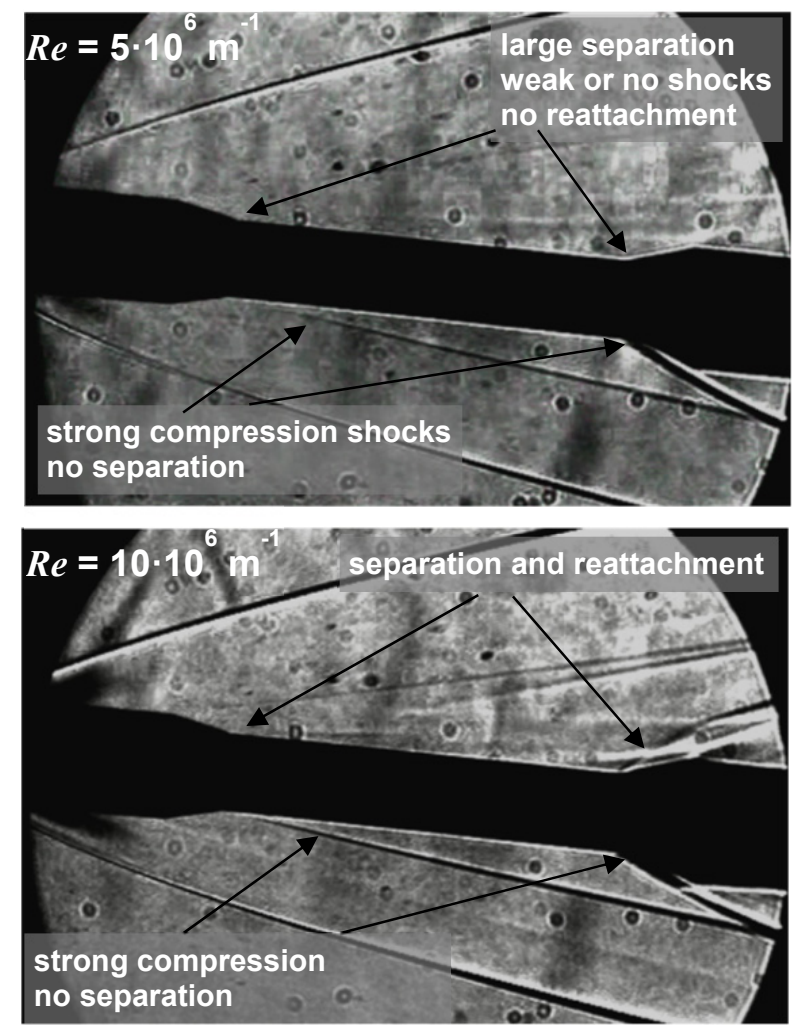

Figure 7: Schlieren images for low (left) and high (right) Reynolds number conditions and $\alpha=-5^{\circ}$
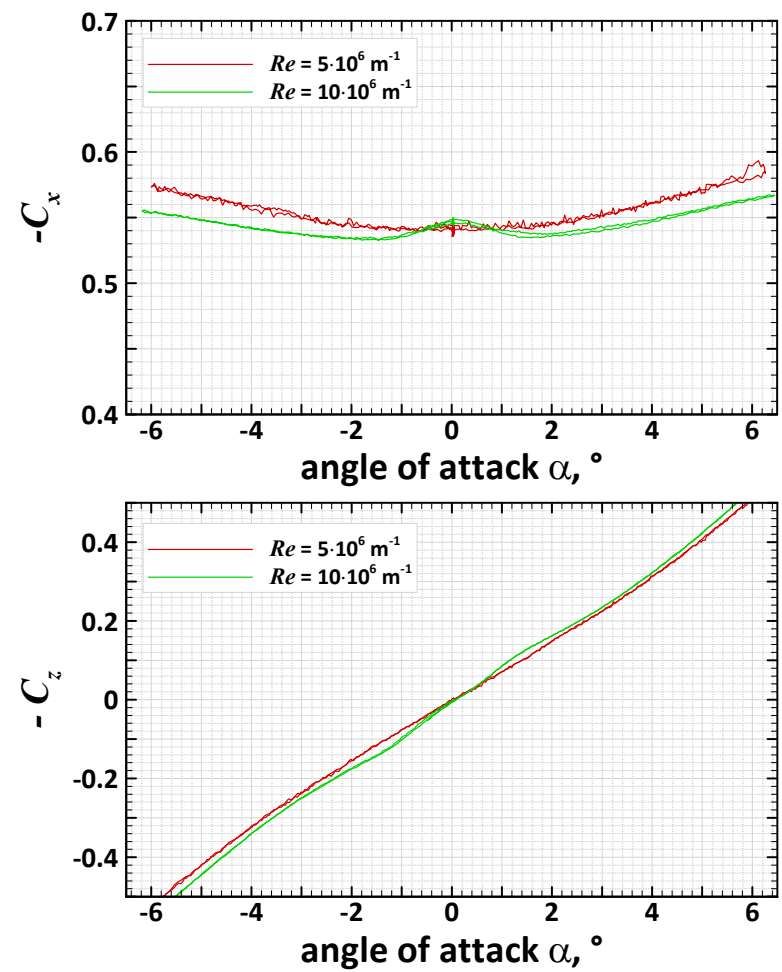

Figure 8: Axial (top) and normal (bottom) force coefficients for cases without retro rocket injection 


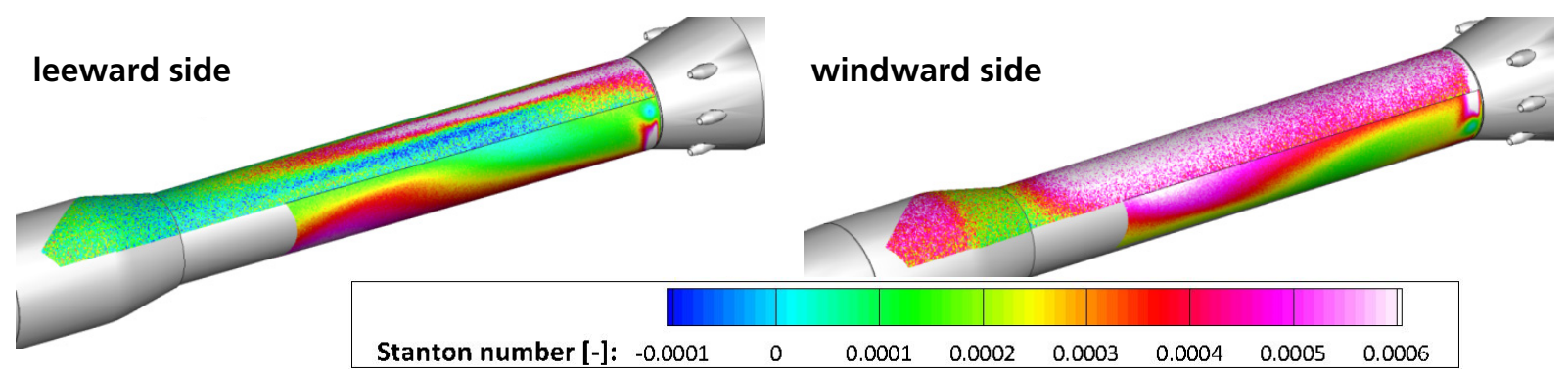

Figure 9: Stanton number distributions for condition WT 1 with $\operatorname{Re}=5 \cdot 10^{6} \mathrm{~m}^{-1}$ and $\alpha=5^{\circ}$

\section{B. Cases with retro rocket injection}

\section{Influence of injection pressure ratio}

Figure 10 shows the flowfield for condition WT 1 and different injection pressure ratios. In both cases, the retro rocket jets cause the flow around the upper stage to almost completely separate. The separation extends far upstream up to the point where the ogive of the hammerhead transitions into the cylindrical part. In case of the higher pressure ratio, the lateral extent of the separation bubble is much greater with its edge being close to the vehicle bow shock and interacting with it. When the pressure ratio is increased during the test run, at certain points, sudden changes in the flow structure occur, at which the separation becomes significantly larger and the angle of the bow shock gets steeper. When this happens, the structure of the retro rocket jets changes as well. The jets detach more from the upper stage surface and the Mach disc marking its end moves further upstream. Also the region, where the Mach disc interacts with the vehicle surface protrudes upstream.

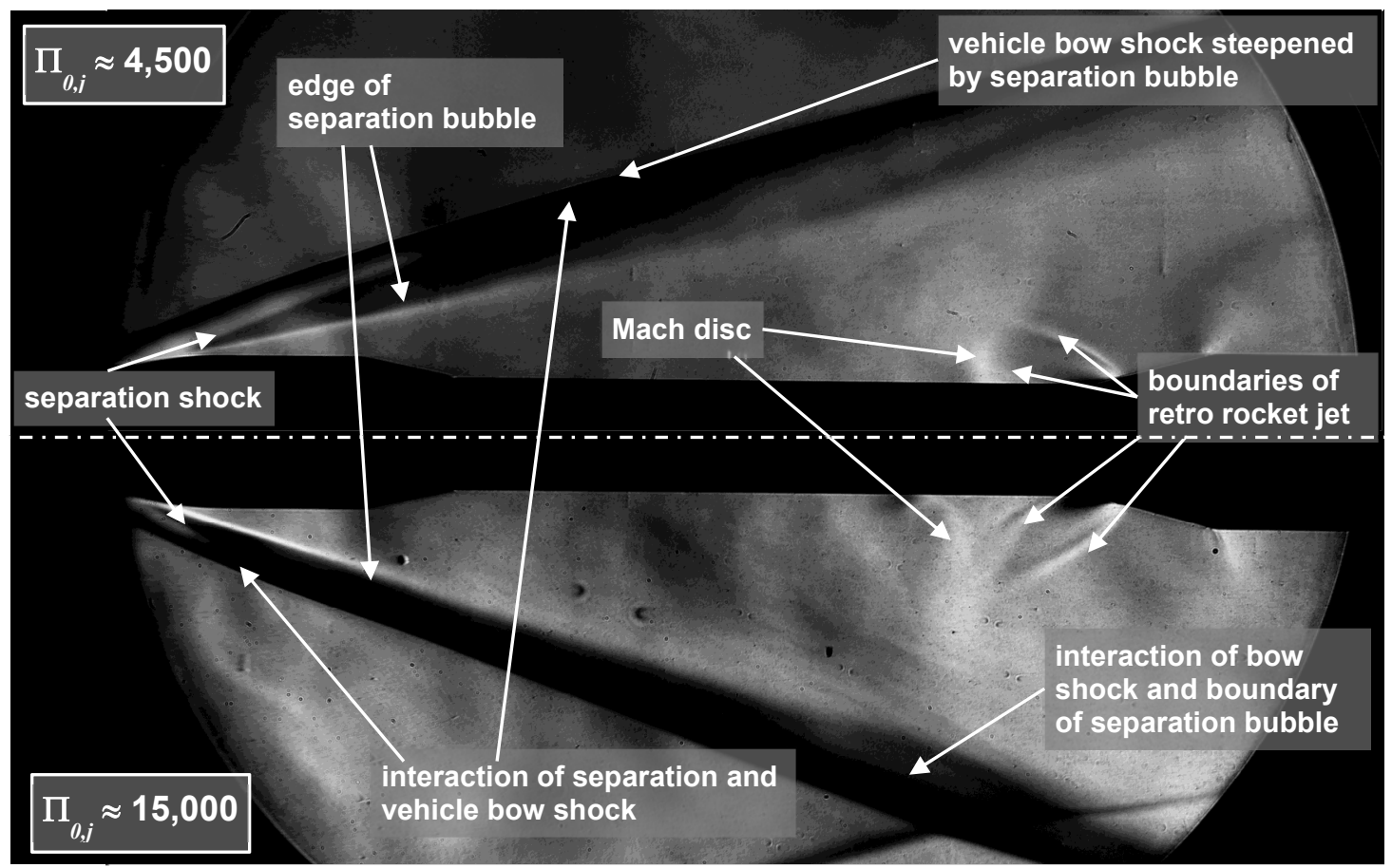

Figure 10: Schlieren image of the flowfield for condition WT 1 and different injection pressure ratios

Figure 11 shows the Stanton number distributions of these two cases. These images give a clearer image of how the retro rocket jets interact with vehicle surface, where the jets cause an upstream flow along the upper stage. As the retro rocket flow is very cold due to the expansion in the nozzle, with the static temperature around $\mathrm{T}_{\text {ex }, \mathrm{j}}=81 \mathrm{~K}$ in the nozzle exit plane and dropping even further by after-nozzle expansion, the surface temperature drops significantly, at the points where the jets hit the upper stage surface. This is indicated by the three spots with lower Stanton number directly upstream of the flare. Furthermore, the interaction of the jets with each other seem to induce vortices, which in turn cause more of the cold jet flow to be transported towards the surface and generate a further two

8

American Institute of Aeronautics and Astronautics 


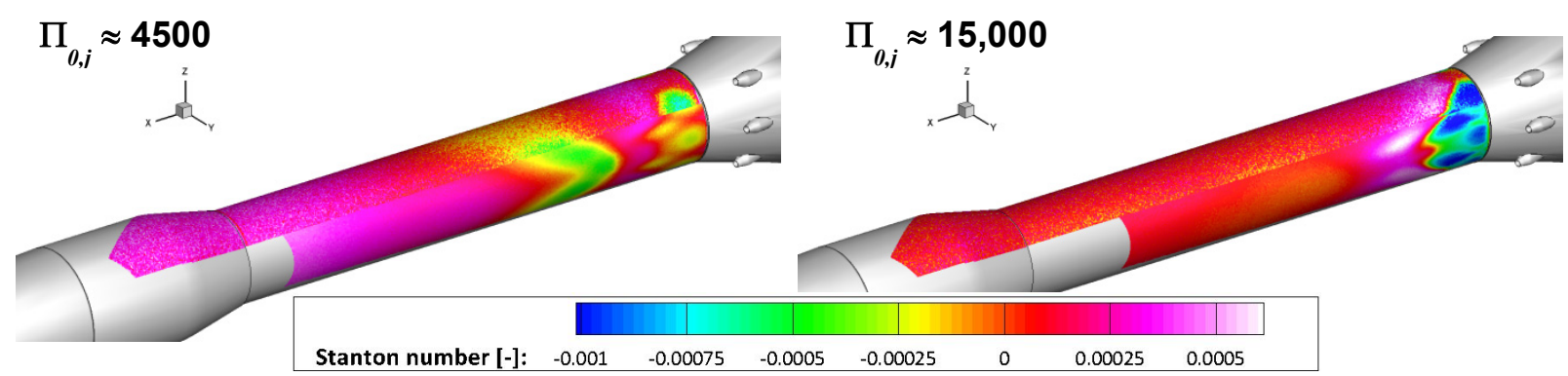

Figure 11: Stanton number distributions for condition WT 1 and different injection pressure ratios

cold spots in between the jets a bit further upstream. It is to expected, that in real flight where the injection gases are very hot, corresponding hot spots will be created. So far, the structure is similar for both pressure ratios, although the patterns are more distinct for the higher pressure ratio and the temperature drop and corresponding decrease in Stanton number in the areas, where the retro rocket jets interact with the surface is much greater. Apparently, this is due to the interaction between the jets being much stronger.

With the higher pressure ratio, this area also has a clear boundary which corresponds to the Schlieren images, i.e. the point at which the Mach disc interacts with the surface. Outside of this area, the Stanton number is rather constant, except for the regions close to the boundaries, which exhibit higher values. This is presumably caused by warmer flow which is led around the boundaries of the retro rocket jets onto the upper stage surface. For the lower injection pressure ratio, this interaction zone is not restricted to the area close to the retro rockets. A further Vshaped area with lower Stanton number is induced further upstream.

The pressure distributions for windtunnel condition WT 1 and the two cases with different injection pressure ratios are shown in Figure 12. As a reference, the case without retro rocket injection is included as well. The pressure along the centreline of both the top and the side are displayed. If the retro rockets are off, the pressure is nearly constant and lower than the freestream static pressure. It is interesting to note that the pressure at the most downstream pressure port, which is closest to the retro rockets, is not affected at all by the injection. The pressure is the same for all cases and there is also no difference in between the side and the top of the model. The retro rockets' effect on the wall pressure is greater, the further you go upstream as the pressure increases quite steadily. Except for the second pressure tab from the

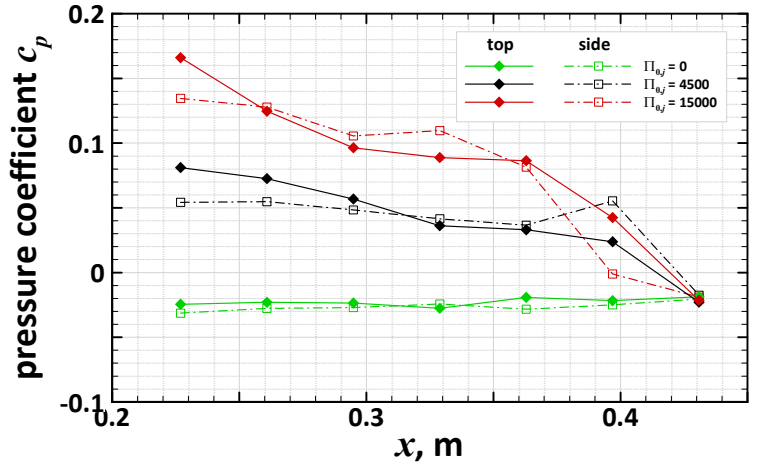

Figure 12: Pressure distribution on upper stage surface for different injection pressure ratios downstream end, the differences between the side and the top are rather small and the curves are quite similar for both $\Pi_{0, j}=4,500$ and 15,000 with a rather constant difference in their pressure levels. At the downstream end, there is a strong pressure rise for $\Pi_{0, j}=4,500$ from the first to the second pressure port and then a decreases to the third. For $\Pi_{0, \mathrm{j}}=15,000$ there is only a small increase in between the first and second pressure tabs, but then a strong increase to the third. This a probably caused by the jet being more attached to the surface for the lower pressure ratio. It also seems to correlate with the location of the additional cold structure visible on the surface in the infrared measurements in this case.

\section{Reynolds number influence}

Figure 13 shows a Schlieren image and Stanton number distribution for condition WT 2 with the higher Reynolds number of $R e=10 \cdot 10^{6} \mathrm{~m}^{-1}$ and retro rocket condition $\mathrm{RR} 1$, resulting in $\Pi_{0, \mathrm{j}} \approx 4,500$. There are no significant differences in the flow topology when compared to the case with the same pressure ratio and $R e=5 \cdot 10^{6} \mathrm{~m}^{-1}($ the upper half of Figure 10). The extent of the separation and the shape of the plume are very similar. Furthermore, the Stanton number shows comparable feature with several cold spots directly upstream of the retro rockets and a Vshaped structure further upstream. Quantitatively, however, the values of the Stanton number on the surface only vary in a much smaller range, i.e. the Stanton number in the areas that are affected by the interaction of the retro rocket plumes with the surface is significantly higher than in the case with the lower Reynolds number and in the remaining parts, it is considerably higher. 

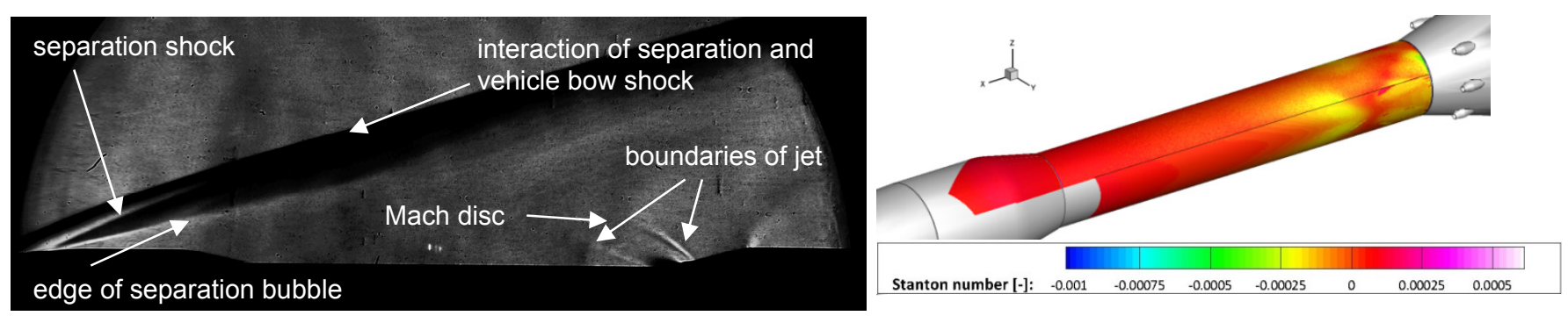

Figure 13: Schlieren image (left) and Stanton number distribution (right) for $R e=10 \cdot 10^{6} \mathrm{~m}^{-1}$ and $\Pi_{0, j}=4,500$

\section{Angle of attack}

The angle of attack was found to have severe influence on the flowfield and the aerodynamic coefficients. Generally, when an angle of attack is applied, the vehicle bow shock on the windward side moves closer to the surface, squeezing the separation bubble while it expands laterally on the leeward side. However, for the configuration that is investigated, these changes do not occur steadily but exhibit sudden changes in the flow structure resulting in jumps in the aerodynamic coefficients. The occurrence of these phenomena is influenced by hysteresis effects. This can be seen by Figure 14 which shows Schlieren images for different angles of attack during the first leg of an $\alpha$-sweep as described in section II, i.e. when the model is moved from $\alpha=0^{\circ}$ to $\alpha=-6^{\circ}$, and the curves of the axial and normal force coefficients for the separate parts of the $\alpha$-sweep are displayed in Figure 15. In the range of $-2^{\circ}>\alpha>-2.5^{\circ}$, interactions between the vehicle bow shock and the edge of the separation bubble can be noted. These result in the vehicle bow shock jumping closer to the surface on the windward side, and the retro rocket plume on the leeward side to not detach from the upper stage surface any more.
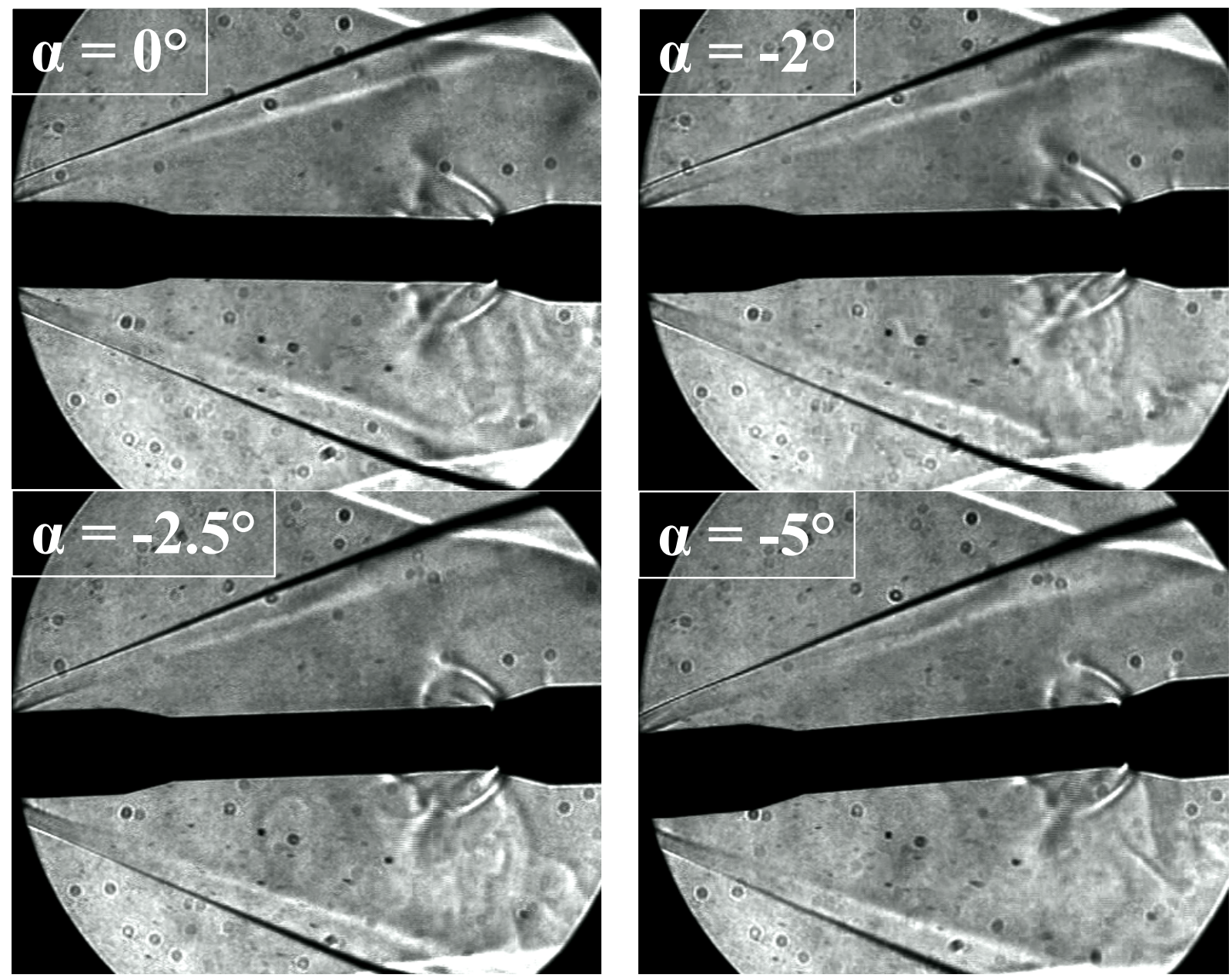

Figure 14: Schlieren images for condition WT 1 with $\Pi_{0, j}=15,000$ and different angles of attack

10

American Institute of Aeronautics and Astronautics 

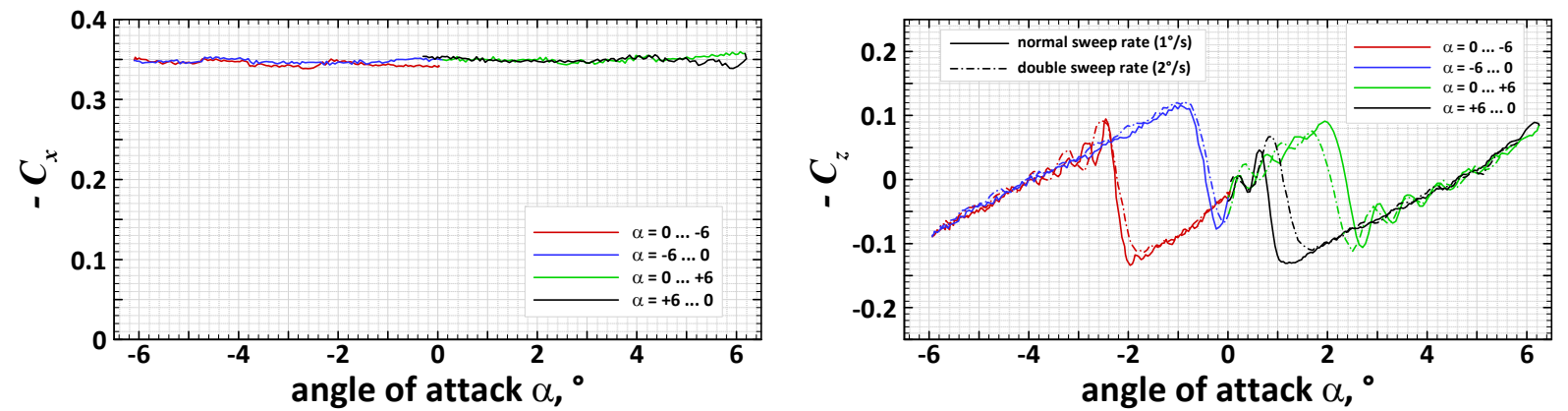

Figure 15: Axial (left) and normal (right) force coefficients for condition WT 1 with $\Pi_{0, j}=15,000$ and different angles of attack

These changes in the flow structure cause the normal force, which first drops when the model is moved out of its zero position towards negative angle of attack, to suddenly jump to a positive value, meaning a direction change of this force. In real flight, the normal force acts like a side force on the launcher. A sudden change in the direction of this force could have severe impact on its flight stability. The vehicle drag, i.e. the axial force coefficient, is not influenced at all by the angle of attack, however.

As already mentioned, this effect is subject to hysteresis, as the points, at which these sudden changes occur, are different depending on whether the model is moved out of its zero position or back towards it. However, the behaviour is symmetric, meaning these changes occur at the same values for negative and positive angles. It is also very well repeatable, and hardly influenced by the sweep rate. For the normal force coefficients in Figure 15, a test run with doubled sweep rate is included as well. Only for positive angles of attack, the points where the sudden changes occur show some minor deviations with the changes occurring a bit sooner at the higher sweep rate. For negative angles, they agree very well. Apart from these jumps, the flowfield is very stable and only changes steadily with the angle of attack and the curves of $C_{z}$ are linear.

The characteristics of the wall pressure at different pressure tabs displayed in Figure 16 give a bit more insight regarding the areas, where these strong changes occur. The curves in the graphs show the most upstream pressure $\operatorname{tab}\left(p_{s t} 1\right)$, the one in the middle $\left(p_{s t} 4\right)$ and the most downstream location $\left(p_{s t} 7\right)$ which is closest to the retro rockets. It shows that the strong change of the normal force at $\alpha \approx-2^{\circ}$ on the first leg is caused by a pressure drop at downstream end on the top, i.e. the windward side. This is remarkable as the Schlieren images did not show significant changes of the jet plume on the windward, but on the leeward side. The pressure at the measurement point in the middle is only moderately affected and decreases quite linearly with both positive and negative angle of attack. At the upstream end, the pressure remains fairly constant for all angles. The same is true for the upstream end at the side of the model but also for the most downstream location, which is not surprising as Figure 12 already showed that the pressure at the tab closest to the retro rockets is not affected at all by the injection. In the middle section of the upper stage, a rather linear pressure drop with both positive and negative angles is present. It is similar to the one at the top but a bit more distinct. While the pressure is equal on the top and the side for $\alpha=0^{\circ}$, it is considerably lower for the maximum angles.
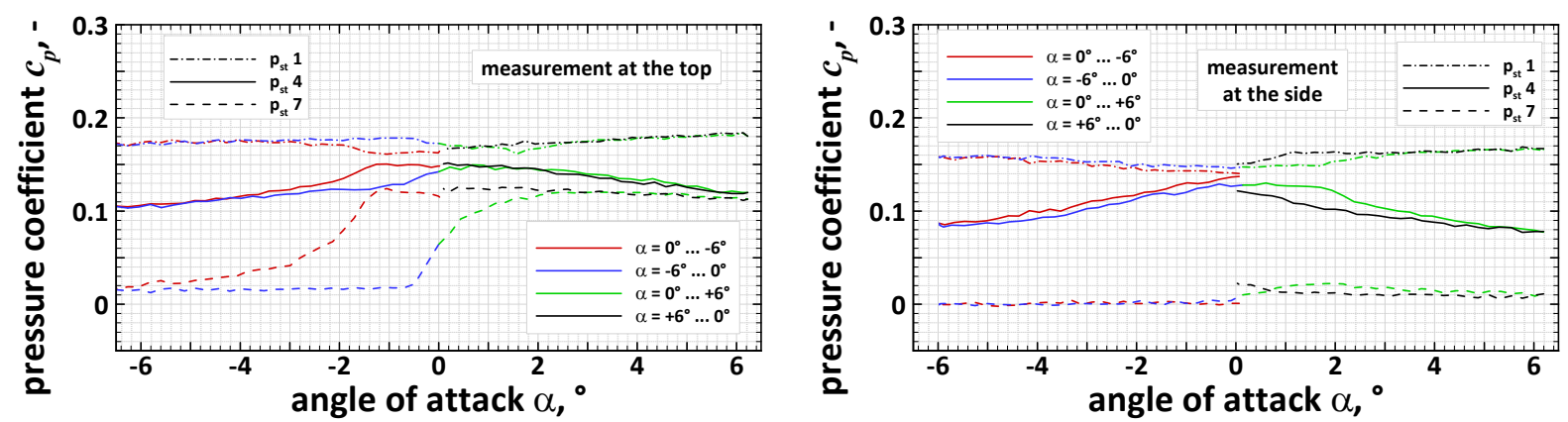

Figure 16: Characteristics of various wall pressure tabs on the top (left) and side (right) of the model for condition WT 1 and $\Pi_{0, j}=15,000$ during an angle of attack sweep 


\section{Influence of injection pressure ratio and Reynolds number during $\alpha$-sweep}

The impact the injection pressure ration and the Reynolds number have on the aerodynamic coefficients during an angle of attack sweep can be seen in Figure 17. The cases without injection are included as a reference. The drag is decreased by the retro rocket injection. Not only does the separation around the upper stage reduce skin friction drag by the freestream, but the plume actually creates a positive axial force on the upper stage by skin friction of the upstream flow inside the separation and by pushing against the flare of the hammerhead and the baseplate by flow entering the cavity in between the two stages. For equal pressure ratios, i.e. $\Pi_{0, \mathrm{j}}=4,500$, the difference in between the two Reynolds numbers is almost similar as in the case without injection. For both cases, a slight increase in drag at both positive and negative angle of attack is noted, as opposed to the case with higher pressure ratio, where it remains constant for all angles.

The behaviour of the flowfield with the lower pressure ratio is much less stable for both Reynolds numbers. There are no distinct points with strong changes of the flow topology and the aerodynamic coefficients, yet the curves are not really steady and continuous as for the cases without injection. Generally, the variation of the normal force with the angle of attack is less than without injection and still rather linear, but there are frequent small fluctuations in the flow structure and corresponding changes in the normal force. Overall, the influence of the Reynolds number is fairly small. The much more influencing factor regarding the retro rockets' impact on the aerodynamic forces is the injection pressure ratio.
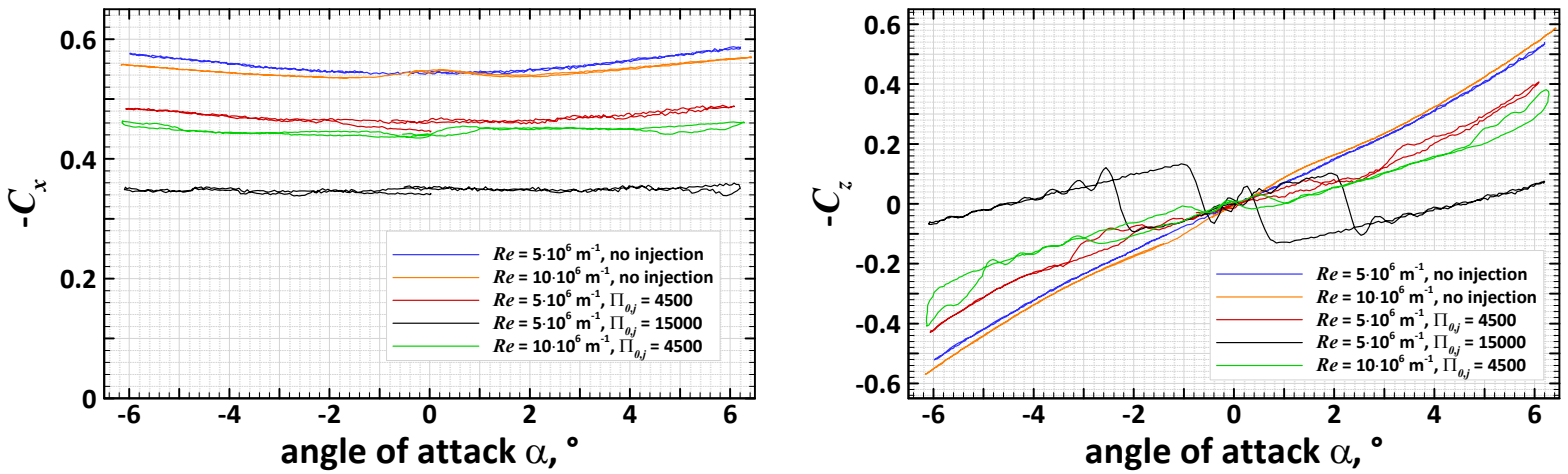

Figure 17: Coefficients of axial (left) and normal (right) forces for different conditions

\section{Conclusion}

An extensive windtunnel campaign was carried out to investigate the effect the plumes of retro rockets have on the aerodynamics of the upper stage of a sequential staged launcher during the separation process. The investigation showed that the plumes of the retro rockets cause significant disturbances in the flowfield surrounding the upper stage, strongly influence the thermal loads on the upper stage surface and can have severe impact on their flight dynamics.

Already at low injection pressure ratios, the flowfield separated almost completely. While variation of the pressure ratio did not have significant impact on the flow topology, it still had strong influence on the aerodynamic forces. The Reynolds number was not found to have a considerable effect. Aerodynamic forces were found to show strong dependency on the angle of attack, as sudden changes of the flow topology occurred at certain angles, which were accompanied by corresponding changes in the normal force. At high injection pressure ratios, theses were so large that the force changed its direction. This might be problematic regarding the stability of the upper stage in real flight.

\section{Acknowledgements}

The authors would like to thank for the funding this work as part of the ESA TRP "Launcher Stage Separation and Plume Interaction Validation", and Neil Murray as the ESA project officer. Further thanks go to the windtunnel team Michael Kosbow, Marco Schmors and Manuel Schorn for the conduction of the experiments. 


\section{References}

Pamadi, B., Pei, J., Pinier, J. T., Holland, S. D., Covell, P. F., and Klopfer, G. H., "Aerodynamic Analyses and Database Development for Ares I Vehicle First-Stage Separation," Journal of Spacecraft and Rockets, vol. 49, Sep. 2012, pp. 864-874.

VEGA - The Small Launcher for Europe, European Space Agency (ESA), 2005.

Paglia, F., Pizzicaroli, A., Lambiase, E., Contini, C., Dumaz, C., Stella, F., Giangi, M., and Barbagallo, D., "Vega launcher aerodynamics at separation of 1st Stage," 43rd AIAA/ASME/SAE/ASEE Joint Propulsion Conference \& Exhibit, Cincinnati, OH, USA: AIAA-2007-5859, 2007.

Genito, M., Paglia, F., Mogavero, A., and Barbagallo, D., "1st Stage Separation Aerodynamics of VEGA Launcher," Proceedings of the 7th European Symposium on Aerothermodynamics for Space Vehicles (ESA SP-692), Brugge, Belgium: 2011.

Zhao, X., Qian, H., Yan, B., and Zhang, W., "Aerodynamic researches on stage separation with two reversal asymmetric jets," 17th AIAA International Space Planes and Hypersonic Systems and Technologies Conference, San Francisco, CA, USA: AIAA-2011-2322, 2011.

Niezgodka, F.-J., Der Hyperschallwindkanal H2K des DLR in Köln-Porz (Stand 2000), German Aerospace Center (DLR), DLR Mitteilung 2001-01, 2001.

Pressure Systems 8400 Users Manual, Pressure Systems Inc., 1991.

Kulite XCQ-080 Series Miniature Pressure Transducer, Datasheet, 2012.

InfraTec, InfraTec ImageIR 8300 Data Sheet, 2014.

Systems, F., ThermaCam SC-3000 Users Manual, Portland, OR: 1999.

Henckels, A., and Gruhn, P., "Study on Aerothermal Effects of Viscous Shock Interaction in Hypersonic Inlets," Proceedings of the Fifth European Symposium on Aerothermodynamics for Space Vehicles, Cologne, Germany: 2005.

Abrall, R., Desideri, J.-A., Glowinski, R., Mallet, M., and Periaux, J., "Proceedings of the INRIAGAMNI/SMAI Workshop on Hypersonic Flows for Reentry Problems, Part II," Antibes, France: Springer, Berlin, 1993, p. 5. 